E4-2004-180

M. I. Shirokov*

\title{
CAUCHY INEQUALITY AND UNCERTAINTY RELATIONS FOR MIXED STATES
}

Submitted to «International Journal of Theoretical Physics»

*E-mail: shirokov@thsun1.jinr.ru 
Неравенства Коши и соотношения неопределенностей для смешанных состояний

Неравенство Коши связывает скалярное произведение двух векторов и их нормы. Исходя из него Шредингер вывел свое соотношение неопределенностей. Указаны другие сходные неравенства, из которых получены соответствующие разные соотношения неопределенностей. Показано, что все они следуют из шредингеровского. Получены два обобщения неравенства Коши для смешанных состояний, описываемых матрицами плотности. Исходя из них для таких состояний выведены два разных обобщения соотношений неопределенностей, отличающиеся от обобщения, известного в литературе. Когда состояния «чистые» (описываются векторами), все три обобщения переходят в шредингеровское соотношение неопределенности. Обсуждены их отличия.

Работа выполнена в Лаборатории теоретической физики им. Н. Н. Боголюбова ОИЯИ

Препринт Объединенного института ядерных исследований. Дубна, 2004

Shirokov M. I.

E4-2004-180

Cauchy Inequality and Uncertainty Relations for Mixed States

Cauchy inequality (CI) relates scalar products of two vectors and their norms. I point out other similar inequalities (SI). Starting with CI Schrödinger derived his uncertainty relation (UR). By using SI other various UR can be obtained. It is shown that they follow from the Schrödinger UR. Two generalizations of CI are obtained for mixed states described by density matrices. Using them two generalizations of UR for mixed states are derived. Both differ from the UR generalization known from the literature. The discussion of these generalizations is given.

The investigation has been performed at the Bogoliubov Laboratory of Theoretical Physics, JINR. 


\section{INTRODUCTION}

Heisenberg uncertainty relation (HUR) $\sigma_{x}^{2} \sigma_{y}^{2} \geqslant \hbar^{2} / 4$ is the most known example of the quantum inequalities which are considered here. It is the corollary of quantum postulates. The main postulate is the description of physical system states by vectors of linear space with a scalar product (e.g., Hilbert space). For two vectors $\alpha_{1}$ and $\alpha_{2}$ one can also derive from these postulates the known CauchyBunyakowskii-Schwarz inequality $\left(\alpha_{1}, \alpha_{1}\right)\left(\alpha_{2}, \alpha_{2}\right) \geqslant\left|\left(\alpha_{1}, \alpha_{2}\right)\right|^{2}$, which will be called the Cauchy inequality $(\mathrm{CI})$.

HUR restricts possible values of dispersions of observables. CI can also be given in a physical sense: it is a premise for the probability interpretation of state vectors, see Sec. 1 below.

Robertson and Schrödinger [6,7] pointed out an uncertainty relation which is more general than HUR. It is usually called the Schrödinger uncertainty relation (SUR), see [2,9,10]. Schrödinger [7] derived SUR starting with CI and then obtained HUR as a particular case of SUR.

Various modifications and generalization of $\mathrm{CI}$ and uncertainty relations (UR) are known. For example, there exist inequalities which contain three and more state vectors or observables, e.g., see $[2,10,8]$. Here we consider CI for two states and UR for two observables, the states being described either by vectors (pure states) or by density matrices (mixed states).

It is pointed out in Sec. 1 that along with CI many similar inequalities (SI) can be written which turn out to be particular cases of CI. Starting with one of these SI one can obtain HUR. From another SI one can derive other useful UR, see Subsec. 1.3.

Two generalizations of CI are obtained in Sec. 2 for the case of two mixed states described by density matrices $W_{1}$ and $W_{2}$. Both turn into CI in the case of pure states. One of them allows one to introduce the notion «the probability to find the mixed state $W_{1}$ in the mixed state $W_{2}$ ».

The generalization of UR for the mixed state $W$ is known (see, e.g., review [2]). Two new generalizations are derived in Sec. 3. All these three generalizations are different consequences of quantum postulates. All turn into the same SUR when $W$ describes a pure state. For further discussion of these generalizations see the Conclusion. 


\section{CAUCHY INEQUALITY IN QUANTUM MECHANICS AND UNCERTAINTY RELATIONS}

1.1. Cauchy inequality (CI) follows from the postulates of the linear space of vectors $\alpha, \beta, \ldots$ with a scalar product $(\alpha, \beta)$ (e.g., see [1,3]). Further, the following postulates are used:

$$
\begin{array}{ll}
\text { a) } & (\alpha, \xi \beta)=\xi(\alpha, \beta), \quad(\xi \alpha, \beta)=\xi^{*}(\alpha, \beta) ; \\
\text { b) } & \left(\alpha, \beta_{1}+\beta_{2}\right)=\left(\alpha, \beta_{1}\right)+\left(\alpha, \beta_{2}\right) ; \\
\text { c) } & (\alpha, \beta)=(\beta, \alpha)^{*} ; \\
\text { d) } & (\alpha, \alpha) \geqslant 0, \quad \forall \alpha .
\end{array}
$$

Here $\xi$ is a complex number. The property $(d)$ must hold for any two vectors $\alpha_{1}$ and $\alpha_{2}$ and for their superposition $\alpha_{1}+\xi \alpha_{2}$ with an arbitrary $\xi$. Let us call this particular property postulate $\left(d_{2}\right)$. Cauchy inequality may be derived from $(a)-\left(d_{2}\right)$ in the following way (e.g., see [3]). It is easy to verify that when $\xi=-\left(\alpha_{2}, \alpha_{1}\right) /\left(\alpha_{2}, \alpha_{2}\right)$ we have

$$
\left(\alpha_{1}+\xi \alpha_{2}, \alpha_{1}+\xi \alpha_{2}\right)=\left(\alpha_{1}, \alpha_{1}\right)-\left|\left(\alpha_{1}, \alpha_{2}\right)\right|^{2} /\left(\alpha_{2}, \alpha_{2}\right) .
$$

As the 1.h.s. of Eq. (1) must be positive we obtain CI

$$
\left|\left(\alpha_{1}, \alpha_{2}\right)\right|^{2} \leqslant\left(\alpha_{1}, \alpha_{1}\right)\left(\alpha_{2}, \alpha_{2}\right) .
$$

The inequality can be rewritten

$$
\left|\left(\alpha_{1}, \alpha_{2}\right)\right|^{2} /\left(\alpha_{1}, \alpha_{1}\right)\left(\alpha_{2}, \alpha_{2}\right) \leqslant 1 .
$$

This form allows us to give the known probability interpretation to the scalar product $\left(\alpha_{1}, \alpha_{2}\right)$ : the 1.h.s. of (3) may be called the probability to find state $\alpha_{1}$ in the state $\alpha_{2}$ because this 1 .h. s. does not exceed 1, being positive (usually one supposes that $\left.\left(\alpha_{1}, \alpha_{1}\right)=\left(\alpha_{2}, \alpha_{2}\right)=1\right)$.

1.2. Schrödinger [7] derived SUR from CI. Dr. O.V. Teryaev called my attention to that his derivation needs some refinement which I shall consider now.

Let $\psi$ be a state vector such that $(\psi, \psi)=1$, and $A$ and $B$ are observables (Hermitian operators). The latter may have different dimensions, e.g., $A$ has the dimension of length while $B$ is momentum. Then vectors $A \psi$ and $B \psi$ have different dimensions and cannot belong to one linear space (their sum is not defined). Meanwhile vectors $\alpha_{1}, \alpha_{2}$ occurring in inequality (2) (which we are going to start with) must have the same dimension, e.g., be dimensionless. Let us assume

$$
\alpha_{i}=d_{i}^{-1} \Delta A_{i} \psi, \quad \Delta A_{i} \equiv A_{i}-\left(\psi, A_{i} \psi\right), \quad i=1,2 .
$$


Here $d_{1}$ and $d_{2}$ are arbitrary constants which have the same dimensions as $A_{1}$ and $A_{2}$, respectively. The defined $\alpha_{1}, \alpha_{2}$ are of the same dimension as $\psi$ (e.g., are dimensionless). Substituting (4) into (2) we get

$$
\left(d_{1}^{-1} d_{2}^{-1}\right)^{2}\left|\left(\psi, \Delta A_{1} \Delta A_{2} \psi\right)\right|^{2}=d_{1}^{-2} d_{2}^{-2}\left(\psi,\left(\Delta A_{1}\right)^{2} \psi\right)\left(\psi,\left(\Delta A_{2}\right)^{2} \psi\right) .
$$

Canceling by $d_{1}^{-2} d_{2}^{-2}$ and using the notation

$$
\sigma_{i}^{2}=\left(\psi,\left(\Delta A_{i}\right)^{2} \psi\right)
$$

we obtain SUR for the dispersions $\sigma_{1}^{2}$ and $\sigma_{2}^{2}$ of the observables $A_{1}, A_{2}$ :

$$
\left|\left(\psi, \Delta A_{1} \Delta A_{2} \psi\right)\right|^{2} \leqslant \sigma_{1}^{2} \sigma_{2}^{2} .
$$

In his derivation Schrödinger omitted the above dimensional constants. In order to derive HUR from inequality (7) Schrödinger (1930) represented 1.h. s. of (7) in the following manner:

$$
\begin{aligned}
& \left(\psi, \Delta A_{1} \Delta A_{2} \psi\right)=(\psi, \hat{R} \psi)+(\psi, i \hat{J} \psi), \\
& \hat{R} \equiv \frac{1}{2}\left\{\Delta A_{1} \Delta A_{2}+\Delta A_{2} \Delta A_{1}\right\}, \quad i \hat{J} \equiv \frac{1}{2}\left[\Delta A_{1} \Delta A_{2}-\Delta A_{2} \Delta A_{1}\right] .
\end{aligned}
$$

The first term in the r.h.s. of Eq. (8) is the average of Hermitian operator $\hat{R}$ and, therefore, is a real number $R$. The second term is imaginary and is equal to $i J, J$ being real ( $\hat{J}$ denotes Hermitian operator). So we have $\left(\psi, \Delta A_{1} \Delta A_{2} \psi\right)=R+i J$, and (7) can be represented as

$$
R^{2}+J^{2} \leqslant \sigma_{1}^{2} \sigma_{2}^{2}
$$

If (10) holds, then we have, of course, $J^{2} \leqslant \sigma_{1}^{2} \sigma_{2}^{2}$, i.e., HUR

$$
\frac{1}{4}\left|\left(\psi,\left[A_{1}, A_{2}\right] \psi\right)\right|^{2} \leqslant \sigma_{1}^{2} \sigma_{2}^{2} .
$$

Let us note that if $\sigma_{1}^{2} \sigma_{2}^{2}=J^{2}$, then it follows from (10) that $R^{2}$ must be zero [7].

1.3. In Subsec. 1.1 CI has been derived from $\left(\alpha_{1}+\xi \alpha_{2}, \alpha_{1}+\xi \alpha_{2}\right) \geqslant 0$ using the special fitting of $\xi: \xi=-\left(\alpha_{2}, \alpha_{1}\right) /\left(\alpha_{2}, \alpha_{2}\right)$. However, the starting inequality must hold for any $\xi$. Various $\xi$ lead to various inequalities similar to CI, let us call them SI. Using substitution (4) one may obtain from SI various UR. A natural question arises: what information these inequalities provide as compared to CI and SUR?

First of all, note that if $\xi$ differs strongly enough from the value $-\left(\alpha_{2}, \alpha_{1}\right) /\left(\alpha_{2}, \alpha_{2}\right)$, then we get from $\left(\alpha_{1}+\xi \alpha_{2}, \alpha_{1}+\xi \alpha_{2}\right)$ no real restrictions for $\left(\alpha_{1}, \alpha_{1}\right),\left(\alpha_{2}, \alpha_{2}\right),\left(\alpha_{1}, \alpha_{2}\right)$. This follows from such easily verifiable identity

$$
\begin{aligned}
& \left(\alpha_{1}+\xi \alpha_{2}, \alpha_{1}+\xi \alpha_{2}\right)=\left(\alpha_{1}, \alpha_{1}\right)-\left(r^{2}+j^{2}\right) /\left(\alpha_{2}, \alpha_{2}\right) \\
& +\left(\alpha_{2}, \alpha_{2}\right)\left\{\rho+r /\left(\alpha_{2}, \alpha_{2}\right)\right\}^{2}+\left(\alpha_{2}, \alpha_{2}\right)\left\{\eta-j /\left(\alpha_{2}, \alpha_{2}\right)\right\}^{2} .
\end{aligned}
$$


Here $\xi=\rho+i \eta$ and

$$
r=\operatorname{Re}\left(\alpha_{1}, \alpha_{2}\right), \quad j=\operatorname{Im}\left(\alpha_{1}, \alpha_{2}\right) .
$$

Indeed if the curly brackets in the r.h.s. of Eq. (12) are large enough, then the positivity of $\left(\alpha_{1}+\xi \alpha_{2}, \alpha_{1}+\xi \alpha_{2}\right)$ is ensured at any $\left(\alpha_{1}, \alpha_{1}\right),\left(\alpha_{2}, \alpha_{2}\right),\left(\alpha_{1}, \alpha_{2}\right)$.

Now let us discuss two examples of SI which give nontrivial inequalities of physical interest. At first, consider the case $\xi=i \eta$, i.e. $\rho=0$. Then Eq. (12) turns into

$$
\left(\alpha_{1}+i \eta \alpha_{2}, \alpha_{1}+i \eta \alpha_{2}\right)=\left(\alpha_{1}, \alpha_{1}\right)-j^{2} /\left(\alpha_{2}, \alpha_{2}\right)+\left(\alpha_{2}, \alpha_{2}\right)\left\{\eta-j /\left(\alpha_{2}, \alpha_{2}\right)\right\} .
$$

If $\eta-j /\left(\alpha_{2}, \alpha_{2}\right)=0$, then it follows from $\left(\alpha_{1}+i \eta \alpha_{2}, \alpha_{1}+i \eta \alpha_{2}\right) \geqslant 0$ that

$$
\left(\alpha_{1}, \alpha_{1}\right)\left(\alpha_{2}, \alpha_{2}\right)-j^{2} \geqslant 0, \quad j=\operatorname{Im}\left(\alpha_{1}, \alpha_{2}\right) .
$$

Inequality (15) is of physical interest because one can obtain from it the HUR, see (11), using substitution (4) (note that $\operatorname{Im}\left(\psi, \Delta A_{1} \Delta A_{2} \psi\right.$ ) has been denoted by $J$ in Subsec. 1.2).

Similarly one may treat another particular case: $\xi=\rho$, i.e. $\eta=0$. Then one gets instead of (15)

$$
\left(\alpha_{1}, \alpha_{1}\right)\left(\alpha_{2}, \alpha_{2}\right)-r^{2} \geqslant 0, \quad r=\operatorname{Re}\left(\alpha_{1}, \alpha_{2}\right),
$$

and instead of HUR the inequality

$$
\frac{1}{4}\left|\left(\psi,\left\{\Delta A_{1} \Delta A_{2}+\Delta A_{2} \Delta A_{1}\right\} \psi\right)\right|^{2} \leqslant \sigma_{1}^{2} \sigma_{2}^{2} .
$$

This UR means that the product of dispersions may be restricted from below also in the case when observables $A_{1}$ and $A_{2}$ commute and HUR turns into the inequality $\sigma_{1}^{2} \sigma_{2}^{2} \geqslant 0$ which is trivial: dispersions are positive by definition, see Eq. (6).

Let us stress that $\mathrm{CI}$ is not only a necessary consequence of the postulate $\left(d_{2}\right)$ but also is sufficient: the postulate $\left(d_{2}\right)$, i.e. $\left(\alpha_{1}+\xi \alpha_{2}, \alpha_{1}+\xi \alpha_{2}\right) \geqslant 0$, follows from CI because squares of the curly brackets in Eq. (12) are positive at any $\xi$. Meanwhile SI are not sufficient (being necessary, of course). For example, let (15) holds. Then the r.h.s. of Eq. (12) may turn out to be negative because it contains besides nonnegative contributions also the negative one $-r^{2} /\left(\alpha_{2}, \alpha_{2}\right)$.

So CI is equivalent to $\left(d_{2}\right)$ and may replace it. Another proof of this equivalency is presented in [8], Subsec. 2.1, the case $n=2$.

1.4. The relation of SUR following from CI to other UR following from SI (e.g., see (11) and (16)) can be formulated using a physical language: SUR is the most restrictive inequality for dispersions. Other UR, e.g., (11) and (16), 
follow from SUR. For example, the region of possible values of $\sigma_{1}^{2} \sigma_{2}^{2}$ which is allowed by (11) is greater than the region allowed by SUR. In particular, when $A_{1}$ and $A_{2}$ commute inequality (11) turns into trivial one $\sigma_{1}^{2} \sigma_{2}^{2} \geqslant 0$, while SUR, see (10), shows that in this case $\sigma_{1}^{2} \sigma_{2}^{2}$ must be larger than a nonzero (generally) quantity $R^{2}$. However, HUR is simpler than SUR and, therefore, may be useful. For example, if $\left[A_{1}, A_{2}\right]$ is equal to a number $i c$, then for any normalizable $\psi$ the product $\sigma_{1}^{2} \sigma_{2}^{2}$ is restricted from below by the known constant: $c^{2} / 4 \leqslant \sigma_{1}^{2} \sigma_{2}^{2}$.

\section{CAUCHY INEQUALITIES FOR DENSITY MATRICES}

Till now physical system states were supposed to be described by vectors of linear space (e.g, Hilbert space). In this section the states are described by density matrices. Two various inequalities for two density matrices will be obtained which generalize CI, see (2).

2.1. The derivation of the first inequality uses the following definition of the density matrices $W_{1}$ and $W_{2}$ :

$$
W_{i}=\sum_{m} p_{m}^{(i)}\left|\omega_{m}^{(i)}\right\rangle\left\langle\omega_{m}^{(i)}\right|, \quad p_{m}^{(i)}>0, \quad i=1,2,
$$

where $\omega_{m}$ are state vectors and $p_{m}$ are their weights. The orthogonality of $\omega_{m}$ is not supposed. I do not suppose also that $\left(\omega_{m}, \omega_{m}\right)=1$ and that $\operatorname{Sp} W_{i}=1$, as is assumed by Messiah [5, v. 1, ch. VIII. 21]. Such a general definition of $W_{i}$ will be needed below in Sec. 3. One has

$$
\begin{aligned}
& \operatorname{Sp} W_{i}=\sum_{m} p_{m}^{(i)}\left\langle\omega_{m}^{(i)} \mid \omega_{m}^{(i)}\right\rangle, \\
& \operatorname{Sp} W_{1} W_{2}=\sum_{m, n} p_{m}^{(1)} p_{n}^{(2)}\left|\left\langle\omega_{m}^{(1)} \mid \omega_{n}^{(2)}\right\rangle\right|^{2} .
\end{aligned}
$$

Taking into account CI

$$
\left|\left\langle\omega_{m}^{(1)} \mid \omega_{n}^{(2)}\right\rangle\right|^{2} \leqslant\left\langle\omega_{m}^{(1)} \mid \omega_{m}^{(1)}\right\rangle\left\langle\omega_{n}^{(2)} \mid \omega_{n}^{(2)}\right\rangle
$$

and Eq. (18) one gets

$$
\mathrm{Sp} W_{1} W_{2} \leqslant \mathrm{Sp} W_{1} \mathrm{Sp} W_{2} .
$$

Equality in (20) is attained when vectors $\omega_{m}^{(1)}$ are parallel to vectors $\omega_{n}^{(2)}$, $\forall m, n$. In particular, all $\omega_{m}^{(1)}$ must be parallel to one vector, e.g., $\omega_{1}^{(2)}$. This means that vectors $\omega_{m}^{(1)}$ must be parallel to each other: $\omega_{m}^{(1)}\left\|\omega_{m}^{(1)}\right\| \omega_{m}^{(1)} \| \ldots$ Analogously $\omega_{n}^{(2)}$ must be parallel pairwise. However, $W_{1}$ and $W_{2}$ then describe pure states, otherwise one has

$$
0 \leqslant \mathrm{Sp} W_{1} W_{2}<\mathrm{Sp} W_{1} \mathrm{Sp} W_{2}
$$


(the positivity of $\mathrm{Sp} W_{1} W_{2}$ follows from Eq. (19)). So the ratio $\mathrm{Sp} W_{1} W_{2} / \mathrm{Sp} W_{1} \mathrm{Sp} W_{2}$ being positive cannot be equal to unity if both states $W_{1}$ and $W_{2}$ are mixed. This circumstance does not allow one to treat the ratio as «the probability to find the state $W_{1}$ in the state $W_{2}$ ». Indeed, it is natural to expect that such a probability is unity when $W_{1}=W_{2}$ but actually we have $\operatorname{Sp}\left(W_{i}\right)^{2} /\left(\operatorname{Sp} W_{i}\right)^{2}<1$ for a mixed state $W_{i}$.

In the particular case of pure states $W_{i}=\left|\alpha_{i}\right\rangle\left\langle\alpha_{i}\right|$ inequality (20) turns into CI, see (2). So (20) may be considered as the generalization of CI.

2.2. To obtain another inequality for $W_{1}$ and $W_{2}$, let us consider the linear space of Hermitian matrices and their superpositions $Z$ with complex coefficients. The scalar product of elements $Z_{1}$ and $Z_{2}$ (vectors) of the space is defined as the number $\operatorname{Sp} Z_{1}^{\dagger} Z_{2}$ († means Hermitian conjugation). One can verify that the product has the properties

$$
\begin{aligned}
& \operatorname{Sp} Z_{1}^{\dagger} Z_{2}=\left(\operatorname{Sp} Z_{2}^{\dagger} Z_{1}\right)^{*}, \\
& \operatorname{Sp} Z^{\dagger} Z \geqslant 0,
\end{aligned}
$$

i.e., postulates $(c)$ and $(d)$ (see Sec. 1) hold. Let us stress that the matrices $Z$ play the role of vectors of the space.

Let us substitute in inequality (22) the superposition $Z=\sum_{i} \mu_{i} W_{i}$, $i=1,2, \ldots, n$, where $W_{i}$ are (Hermitian) density matrices and $\mu_{i}$ may be any complex numbers. One has

$$
\operatorname{Sp} Z^{\dagger} Z=\sum_{i, j} \mu_{i}^{*} \mu_{j} \operatorname{Sp} W_{i} W_{j}
$$

The r.h.s. of Eq. (23) is of the form $\mu^{\dagger} M \mu=\sum_{i, j} \mu_{i}^{*} M_{i j} \mu_{j}$, where $\mu$ is an arbitrary $n$-vector with components $\mu_{i}$ and $M$ is the $n \times n$ matrix with elements $M_{i j}=\mathrm{Sp} W_{i} W_{j}$. The 1.h.s. of Eq. (23) is nonnegative and, therefore, $M$ must be a positively (more exactly nonnegatively) defined matrix. The necessary and sufficient condition of this property is nonnegativity of all principal minors of $M$, e.g., see [4, ch. 10.4, Theorem 4]. The minors of the first order are equal to $\mathrm{Sp} W_{i}^{2}, i=1,2, \ldots, n$ and they are nonnegative, see Eq. (19). The nonnegativity of the second order minors gives in particular

$$
\left[\operatorname{Sp}\left(W_{1} W_{2}\right)\right]^{2} \leqslant \operatorname{Sp} W_{1}^{2} \operatorname{Sp} W_{2}^{2}
$$

or

$$
0 \leqslant\left[\operatorname{Sp}\left(W_{1} W_{2}\right)\right]^{2} / \operatorname{Sp} W_{1}^{2} \operatorname{Sp} W_{2}^{2} \leqslant 1 .
$$

When $W_{i}=\left|\alpha_{i}\right\rangle\left\langle\alpha_{i}\right|$ inequality (24) turns into «squared» CI

$$
\left|\left(\alpha_{1}, \alpha_{2}\right)\right|^{4} \leqslant\left(\alpha_{1}, \alpha_{1}\right)^{2}\left(\alpha_{2}, \alpha_{2}\right)^{2}
$$

which is equivalent to $\mathrm{CI}$ (see (2)) itself. 
Unlike the above discussed ratio $\mathrm{Sp}\left(W_{1} W_{2}\right) / \mathrm{Sp} W_{1} \mathrm{Sp} W_{2}$ the ratio $\left[\mathrm{Sp}\left(W_{1} W_{2}\right)\right]^{2} / \mathrm{Sp} W_{1}^{2} \mathrm{Sp} W_{2}^{2}$ occurring in (25) turns into unity if $W_{1}=W_{2}$ (even if the state is mixed). This allows one to suggest the notion «the probability $\rho_{12}$ to find the state $W_{1}$ in the state $W_{2}$ » defining it as

$$
\rho_{12}=\operatorname{Sp}\left(W_{1} W_{2}\right) /\left[\operatorname{Sp} W_{1}^{2} \mathrm{Sp} W_{2}^{2}\right]^{1 / 2} .
$$

This quantity turns into the known probability $\left|\left(\alpha_{1}, \alpha_{2}\right)\right|^{2} /\left(\alpha_{1}, \alpha_{1}\right)\left(\alpha_{2}, \alpha_{2}\right)$ when $W_{1}=\left|\alpha_{1}\right\rangle\left\langle\alpha_{1}\right|$ and $W_{2}=\left|\alpha_{2}\right\rangle\left\langle\alpha_{2}\right|$, see Subsec. 1.1.

\section{UNCERTAINTY RELATIONS FOR MIXED STATES}

In Sec. 1, uncertainty relations for pure states were considered. Here I shall deal with various extensions of UR for the case of a mixed state described by a density matrix. I call them mixed state uncertainty relations (MUR).

3.1. The first extension MUR 1 follows from the generalization (20) of CI to the case of mixed states. Consider such density matrices

$$
\begin{aligned}
& W_{i}=\Delta A_{i} W \Delta A_{i}, \quad \Delta A_{i} \equiv A_{i}-\operatorname{Sp} W A_{i}, \quad i=1,2, \\
& W=\sum_{m} p_{m}\left|\omega_{m}\right\rangle\left\langle\omega_{m}\right|
\end{aligned}
$$

(cf. Eq. (4), dimension constants $d_{i}$ are omitted here). I assume that $\left(\omega_{m}, \omega_{m}\right)=$ 1 and $\mathrm{Sp} W=1$, see [5, ch. VIII. 21]. However, the vectors $\Delta A_{i} \omega_{m}$ are not then normalized. Density matrices $W_{1}$ and $W_{2}$ defined by Eq. (27)

$$
W_{i}=\sum_{m} p_{m}\left|\Delta A_{i} \omega_{m}\right\rangle\left\langle\Delta A_{i} \omega_{m}\right|
$$

are particular cases of those used in Sec. 2, Eq. (17). The spurs of $W_{1}$ and $W_{2}$ are not supposed to be equal to unity.

Substituting $W_{i}$ defined by Eq. (27) in inequality (20) one obtains

$$
\operatorname{Sp} \Delta A_{1} W \Delta A_{1} \Delta A_{2} W \Delta A_{2}<\operatorname{Sp}\left(\Delta A_{1}\right)^{2} W \operatorname{Sp}\left(\Delta A_{2}\right)^{2} W .
$$

Here $\operatorname{Sp}(\Delta A)^{2} W$ is the dispersion of the observable $A$ in the mixed state $W$. One gets SUR, see (7), when $W$ describes a pure state: $W=|\psi\rangle\langle\psi|$.

Let us obtain from (28) an inequality resembling HUR. For this purpose represent the 1.h.s. of (28) as

$$
\begin{aligned}
& \operatorname{Sp} W \Delta A_{1} \Delta A_{2} W \Delta A_{2} \Delta A_{1}=\operatorname{Sp} W(\hat{R}+i \hat{J}) W(\hat{R}-i \hat{J}) \\
& =\operatorname{Sp} W \hat{R} W \hat{R}+\operatorname{Sp} W \hat{J} W \hat{J}
\end{aligned}
$$


for $\hat{R}$ and $\hat{J}$ see Eq. (9). Using Eq. (27) one can show that $\operatorname{Sp} W \hat{R} W \hat{R}$ and $\mathrm{Sp} W \hat{J} W \hat{J}$ are nonnegative numbers (because $\hat{R}$ and $\hat{J}$ are Hermitian). Let us denote them as $r^{2}$ and $j^{2}$, respectively. Then (28) can be rewritten as

$$
r^{2}+j^{2} \leqslant \sigma_{1}^{2} \sigma_{2}^{2} .
$$

Inequality (30) resembles (10) but has different positive numbers in its 1.h. s.

If (30) holds, then, of course, $j^{2} \leqslant \sigma_{1}^{2} \sigma_{2}^{2}, j^{2} \equiv \operatorname{Sp} W \hat{J} W \hat{J}$. If the commutator $\left[A_{1}, A_{2}\right]$ is equal to the number $i c$, then $\hat{J}=c / 2$ and $j^{2}=\frac{1}{4} c^{2} \mathrm{Sp} W^{2}$. One obtains the inequality resembling HUR

$$
\frac{1}{4} c^{2} \operatorname{Sp}\left(W^{2}\right) \leqslant \sigma_{1}^{2} \sigma_{2}^{2}
$$

3.2. Using substitution (27) one obtains from another generalization (24) of CI the following extension of UR:

$$
\left[\operatorname{Sp} \Delta A_{1} W \Delta A_{1} \Delta A_{2} W \Delta A_{2}\right]^{2} \leqslant\left[\operatorname{Sp}\left(\Delta A_{1} W \Delta A_{1}\right)^{2}\right]\left[\operatorname{Sp}\left(\Delta A_{2} W \Delta A_{2}\right)^{2}\right] .
$$

This extension, MUR 2, is more cumbersome than the previous one. In the particular case $W=|\psi\rangle\langle\psi|$, inequality (32) turns into the «squared» SUR

$$
\left|\left(\psi, \Delta A_{1} \Delta A_{2} \psi\right)\right|^{4} \leqslant \sigma_{1}^{4} \sigma_{2}^{4},
$$

which is equivalent to SUR, see (7).

Inequality (32) does not contain dispersions. However, using the inequalities $\operatorname{Sp}\left(W_{i}^{2}\right) \leqslant\left[\operatorname{Sp} W_{i}\right]^{2}$ for the density matrices $W_{i}$, see Eq. (27), one obtains from (32) the relaxed inequality

$$
\left[\operatorname{Sp} \Delta A_{1} W \Delta A_{1} \Delta A_{2} W \Delta A_{2}\right]^{2} \leqslant \sigma_{1}^{4} \sigma_{2}^{4}
$$

which contains dispersions. It coincides with the «squared» inequality (28), i.e., with (28) itself. As far as the relaxation of (32) coincides with (28), one may conclude that inequality (32) itself is more restrictive than (28).

3.3. Let us set forth the derivation of the known generalization of SUR for a mixed state, cf. [1, ch. II. 6], [2, ch. 2]. I call it MUR 3.

Consider linear space of Hermitian matrices $A_{i}$ (observables) and their superpositions $Z=\sum_{i} \mu_{i} A_{i}$ with complex coefficients $\mu_{i}$. The matrices play the role of vectors of this space. Their scalar product $\left(Z_{1}, Z_{2}\right)$ is defined in a different way than in Subsec. 2.2.

$$
\left(Z_{1}, Z_{2}\right) \equiv \operatorname{Sp} W Z_{1}^{\dagger} Z_{2}
$$

It has the properties $\left(Z_{1}, Z_{2}\right)=\left(Z_{2}, Z_{1}\right)^{*}$ and

$$
(Z, Z)=\operatorname{Sp} W Z^{\dagger} Z \geqslant 0, \quad \forall Z \text {. }
$$


Inserting $Z=\sum_{i} \mu_{i} \Delta A_{i}, i=1,2$ into (34) one gets

$$
\operatorname{Sp} W Z^{\dagger} Z=\sum_{i, j} \mu_{i}^{*} \mu_{j} \operatorname{Sp} W \Delta A_{i} \Delta A_{j}
$$

Due to (34) the r. h.s. of (35) must be nonnegative. The necessary and sufficient condition of this property is the nonnegativity of principle minors of the $2 \times 2$ matrix with the elements $\operatorname{Sp} W \Delta A_{i} \Delta A_{j}$, cf. Subsec. 2.2. In particular, the nonnegativity of the second order minor gives the desired MUR

$$
\left|\operatorname{Sp} W \Delta A_{1} \Delta A_{2}\right|^{2} \leqslant \operatorname{Sp}\left(\Delta A_{1}\right)^{2} W \operatorname{Sp}\left(\Delta A_{2}\right)^{2} W
$$

(the property $\operatorname{Sp} \Delta A_{1} W \Delta A_{2}=\left(\operatorname{Sp} \Delta A_{2} W \Delta A_{1}\right)^{*}$ was used). Inequality (36) turns into SUR, see (7) if $W=|\psi\rangle\langle\psi|$.

The obtained MUR can be represented as

$$
R^{2}+J^{2} \leqslant \sigma_{1}^{2} \sigma_{2}^{2} ; \quad R=\operatorname{Sp} W \hat{R}, \quad J=\operatorname{Sp} W \hat{J}
$$

for $\hat{R}$ and $\hat{J}$, see Eq. (9) (one must repeat the computations performed in Subsec. 1.2 substituting $\operatorname{Sp} W \ldots$ for $(\psi, \ldots \psi)$ ). Inequality (37) is analogous to (30). If $\left[A_{1}, A_{2}\right]$ is equal to the number $i c$, one obtains from (37) omitting $R^{2}$

$$
\frac{1}{4} c^{2}(\operatorname{Sp} W)^{2} \leqslant \sigma_{1}^{2} \sigma_{2}^{2}
$$

This inequality coincides with HUR for a normalized pure state, i.e., $c^{2} / 4 \leqslant \sigma_{1}^{2} \sigma_{2}^{2}$ if the mixed state $W$ is also normalized: $\operatorname{Sp} W=1$. As compared to (31) it is more restrictive because $\operatorname{Sp}\left(W^{2}\right) \leqslant(\operatorname{Sp} W)^{2}=1$.

The obtained inequalities MUR 1 (28); MUR 2 (32), and MUR 3 (36), will be discussed in Conclusion.

\section{CONCLUSION}

The known Cauchy inequality (CI) follows from postulates of linear space of state vectors. Starting with CI Schrödinger [7] derived the uncertainty relation, named here SUR.

Besides CI other similar inequalities (SI) follow from the mentioned postulates (for their definition see Sec. 1). They also are of interest because they allow one to obtain some UR, e.g., Heisenberg uncertainty relation (HUR). However, these UR are less restrictive (informative) than SUR, being special cases of SUR.

All UR have a physical sense giving restrictions on dispersions of observables. Cauchy inequality also may be given a physical sense: it is the premise of the known probability interpretation of state vectors, see Subsec. 1.1. 
In Sec. 1, CI and UR for pure states (described by vectors) are discussed. Two generalizations of CI to the case of mixed states (described by density matrices) are obtained in Sec. 2. Both turn into the usual CI when the states are pure.

Starting with these generalizations of CI I have derived in Sec. 3 two extensions of UR for mixed states (named MUR 1 and MUR 2). This was done by means of the approach which Schrödinger [7] used for UR derivation from $\mathrm{CI}$ in the case of pure states. The derivation of the extension of UR for mixed states which is known in the literature (named MUR 3), does not use any CI, see Subsec. 3.3.

So starting with the quantum postulates three UR for mixed states can be obtained. All are necessary corollaries of the postulates. The corollaries are different; e.g., MUR 1 and MUR 3 contain dispersions of observables while MUR 2 does not; the 1.h. s. of MUR 3, see (36), contains $\operatorname{Sp} \Delta A_{1} W \Delta A_{2}$ which is linear in $W$ while the 1.h.s. of MUR 1, see (28), and MUR 2, see (32), contain Spur which quadratically depends upon $W$.

All these UR may be called generalizations of UR for mixed states because they turn into the same SUR when the state is pure.

It was shown in Sec. 1 that SUR is the most restrictive UR in the case of pure states. When the states are mixed MUR 3 seems to be more restrictive than MUR 1: compare (31) with (38). It was also concluded in Subsec. 3.2 that MUR 2 is more restrictive than MUR 1.

One may prefer MUR 3 because it has the following merit: quantities entering it have the known physical sense: $\sigma_{i}^{2}$ are dispersions, and $\operatorname{Sp} W \hat{R}$ and $\operatorname{Sp} W \hat{J}$ are averages of the observables (Hermitian operators) $\hat{R}$ and $\hat{J}$, see Eq. (9). Meanwhile MUR 2, see (32), does not contain dispersions. Besides, both MUR 1 and MUR 2 contain Spurs which depend on $W$ quadratically. Mathematical means of quantum mechanics allow one to calculate such quantities. However, their physical interpretation is unknown and still must be devised. For example, one may suggest to interpret $\mathrm{Sp} W^{2}$ as a measure of «mixity» of the state $W$ ( $\mathrm{Sp} W^{2}$ being unity when the state is pure).

\section{REFERENCES}

1. Bohm A. Quantum Mechanics: Foundations and Applications. N. Y.: Springer-Verlag, 1986.

2. Dodonov V. V., Man'ko V. I. // Trudy FIAN. M., 1987. V.183. P.1-70 (in Russian).

3. Fano G. Mathematical Methods of Quantum Mechanics. N. Y.: McGraw-Hill, 1971.

4. Gantmakher F. R. The Theory of Matrices. N. Y.: Chelsea, 1960. 
5. Messiah A. Quantum Mechanics. Amsterdam: North Holland, 1961.

6. Robertson H. P. // Phys. Rev. A. 1930. V.35. P. 667.

7. Schrödinger E. Sitzugsber. Preuss Akad. Wiss. 1930. P. 296.

8. Shirokov M. I. JINR Preprint E4-2003-84. Dubna, 2003; http://arXiv.org/abs /quant$\mathrm{ph} / 0404165$.

9. Sukhanov A. D. // Phys. Part. Nucl. 2001. V.32. P. 619.

10. Trifonov D. A. // Eur. Phys. Journ. B. 2002. V. 29. P. 349.

Received on November 19, 2004. 
Корректор T. Е. Попеко

Подписано в печать 30.12.2004.

Формат $60 \times 90 / 16$. Бумага офсетная. Печать офсетная.

Усл. печ. л. 0,93. Уч.-изд. л. 1,35. Тираж 350 экз. Заказ № 54733.

Издательский отдел Объединенного института ядерных исследований 141980, г. Дубна, Московская обл., ул. Жолио-Кюри, 6.

E-mail: publish@pds.jinr.ru www.jinr.ru/publish/ 